\title{
Blood Products Laboratory Testing
}

National Cancer Institute

\section{Source}

National Cancer Institute. Blood Products Laboratory Testing. NCI Thesaurus. Code C113077.

In vitro testing of donor blood for safety prior to use in patients. 